\title{
Zoological Research
}

\section{棕背伯劳羽色多态现象探讨}

\author{
江燕琼 ${ }^{1,2}$ ，唐思贤 1 ， 丁志锋 ${ }^{1,2}$, 胡慧建 ${ }^{2, *}$ \\ （1. 华东师范大学 生命科学学院，上海 200062； 2. 华南濒危动物研究所, 广东 广州 510260）
}

摘要: 羽色多态现象的研究对了解物种的遗传、变异和进化有着重要意义。棕背伯劳 (Lanius schach) 具有 典型的羽色多态现象。结合华南濒危动物研究所馆藏标本和 2005 年 4 月-2007 年 1 月间的野外调查, 分析棕背 伯劳棕色型和黑色型在形态和地理分布上的差异性后, 得到的结果如下: 1) 广东可能存在与棕色型、黑色型相区 别的一种新色型—黑色白边型, 该色型全身以黑色为主, 但具多枚白色飞羽; 2) 棕色型与黑色型各身体量度均 无显著性差异 $(P>0.05)$ ； 3) 野外种群中棕色型为优势色型，黑色型多集中于沿海地区而山区未有发现，黑色白边 型仅在沿海的海丰县被发现。据此认为新色型的出现说明沿海可能是黑色型分化的重要地区, 且色型的分化仍在 进行中。

关键词: 棕背伯劳; 羽色多态型; 身体量度; 种群数量

中图分类号：Q959.7 文献标识码：A＼cjkstart文章编号：0254-5853-(2008)01-0099-04

\section{Analysis on Color Polymorphism of Lanius schach}

\author{
JIANG Yan-qiong ${ }^{1,2}$, TANG Si-xian ${ }^{1}$, DING Zhi-feng ${ }^{1,2}$, HU Hui-jian ${ }^{2, *}$ \\ (1. School of Life Sciences, East China Normal University, Shanghai 200062, China; \\ 2. South China Institute of Endangered Animals, Guangzhou 510260, China)
}

\begin{abstract}
Color polymorphism is important in understanding the heredity, variation and evolution of species. Lanius schach is an example of a bird with color polymorphism. Here, we analyzed the deviation of the body measurements and distribution of different color morphs of Lanius schach by combining the specimen data in the South China Institute of Endangered Animals and the field data in Guangdong Province from April 2005 to January 2007. The results were: 1) A possible new color morph, called white-remiged black morph, which is different from brown morph and black morph by having white flight feathers, was identified and only recorded in Haifeng, Guangdong. 2) There was no obvious deviation in body measurements $(P>0.05)$ for brown morphs and black morphs. 3) Brown morphs are more widely distributed and denser than black morphs, which are mostly distributed near seaside areas and never found in mountainous areas. Therefore we concluded that the new color morph suggested seashore regions to be important for the differentiation of black morphs and continued differentiation.
\end{abstract}

Key words: Lanius schach; Color polymorphism; Body measurements; Population

多态现象 (polymorphism) 是指物种的某个个体 特征存在着多种不同形态共存的现象。鸟类羽毛多 态现象是其中最典型的例子之一。多态现象的概念 最早由 Ford (1940)提出, 认为这是物种的某个个体 特征存在着多种不同形态共存的现象(Galeotti et al, 2003)。Huxley（1955）完善了多态现象的概念, 认 为这是两个或两个以上截然不同的可遗传形态，且 相互之间可杂交和共存(Huxley, 1955)。人们针对鸟
类羽色多态现象的进化机制提出了多种假说, 目前 主要有分化选择假说(disruptive selection hypothesis)

(Mather, 1955)、异类选择假说(apostatic selection hypothesis)(Rohwer \& Paulson, 1987)、非随机性交配 假说 (nonrandom mating hypothesis) (Butcher \& Rohwer , 1989)三种。由于鸟类羽毛进化较快(Endler \& Thery, 1996), 并且羽毛特征被用于物种识别及求 偶展示(Marchetti, 1993; Ford, 1940), 因此, 鸟类羽

收稿日期：2007-10-30; 修回日期：2007-12-18

基金项目：国家自然科学基金(30770311); 广东省科学院台站基金(2007,2008 年度); 广州市政府项目(GZ-ZY[2004]0806)

“通讯作者(Corresponding author), Tel: 020 84191955, E-mail: huhj@gdei.gd.cn

第一作者简介: 江燕琼, 女, 硕士研究生, 主要从事鸟类分子生态学研究, E-mail: jyq1825@126.com 
毛多态现象的研究对于阐明鸟类进化机制具有较 为重要的意义, 但在国内对于鸟类羽色多态现象的 研究鲜见报道(Hu et al, 2007)。

棕背伯劳 (Lanius schach) 隶属于雀形目 (Passeriformes)伯劳科(Laniidae), 在中国南方分布 广泛, 并在部分地区存在羽色多态现象, 即在羽色 上有棕色型、黑色型及过渡色型的分化(Zheng, 1976, 2005)。目前, 对于棕背伯劳的羽色多态现象研究仅 见于色型分类地位研究和对不同色型个体有所描 述 (Zheng, 1976; Zhao, 2001)。对于棕背伯劳各羽色 型的分类还存在争议, 有学者将黑色型列为独立种 黑伯劳 (Lanius fuscatus lesson), 而有些学者认为 它是棕背伯劳的一种色型(Zheng, 1976; Zhao, 2001; Chen et al, 1998)。Zheng (1998) 曾在武汉大学标 本馆发现黑色型飞羽出现白化的个体 (Chen et al, 1998)。我们在广东省海丰县也发现黑色型中有部分 个体出现白色飞羽, 该特征与 Zheng（1998）报道 一致。为更充分了解和掌握棕背伯劳羽色多态现象 的特点, 为进一步开展羽色多态现象研究提供前期 性的研究基础, 我们对华南濒危动物研究所馆藏标 本和广东省野外调查结果进行分析和研究, 结果报 道如下。

\section{1 自然概况}

广东省位于 $20^{\circ} 09^{\prime}-25^{\circ} 31^{\prime} \mathrm{N}, 109^{\circ} 45^{\prime}-$ $117^{\circ} 20^{\prime} \mathrm{E}$, 北依南岭, 南临热带海洋, 整体地势北 高南低, 山脉东西走向。广东属于东亚季风区, 从 北向南分别为中亚热带、南亚热带和热带气候, 是 全国光、热和水资源最丰富的地区之一。气候温暖, 雨量充沛。从北向南, 年平均日照时数由不足 1500 $\mathrm{h}$ 增加到 $2300 \mathrm{~h}$ 以上, 年太阳总辐射量在 4200$5400 \mathrm{MJ} / \mathrm{m}^{2}$ 之间, 年平均气温约为 $19-24^{\circ} \mathrm{C}$ 。全省 平均日照时数为 $1745.8 \mathrm{~h}$ 、年平均气温 $22.3^{\circ} \mathrm{C}$, 年 平均降水量 $1777 \mathrm{~mm}$ 。植被主要以亚热带季雨林区 和亚热带常绿阔叶林区为主, 分别集中分布在中部 和北部地区。

\section{2 方 法}

\section{1 身体量度测量和数理分析}

标本取自华南濒危动物研究所标本馆 $(n=59)$ 。 使用杭州华益工具量具有限公司生产的机械游标 卡尺（300 mm, $0.1 \mathrm{~mm}$ ） 对不同羽色型的棕背伯 劳进行包括体长、尾长、嘴峰长、翼长、跗蹠长等
身体量度进行测量。采用 SPSS11.5 统计分析软件, Kolmogorov-Smirnov 检验数据的正态性和方差同 质性, One-way ANOVA 进行差异检验。标本体重 采用标本标签记录。鉴于黑色白边型标本数量太少 $(n=2)$, 未进行数据统计处理。

\section{2 种群调查方法}

在广东地区境内选取广州、海丰、惠州、南岭、 珠海、番禺、雷州、湛江、肇庆、从化等地 35 个 样点进行野外调查 (图 1)。野外种群数量调查采用 样线法, 样线长度 3-5 km, 从早上 6: 30-9: 00, 下午 15: 30-18: 00, 携带双筒望远镜 $(8 \times 42)$ 和 单筒望远镜 $(30 \times 70)$, 以 $1-2 \mathrm{~km} / \mathrm{h}$ 的速度前进, 记录沿途听到的和看到的鸟类物种。

\section{3 结 果}

\section{1 不同色型形态比较}

3.1.1 新色型特征描述２005-2007 年在海丰共 发现 5 只新色型个体 (简称黑色白边型), 其中获 得标本数为 2 只。现将有关特征描述如下: 额、眼 先、眼、颊、耳羽、垓、喉皆黑色; 头顶至上背暗 灰色, 腰至尾上覆羽土褐色, 肩、尾黑色, 两翅主 要是黑色, 但在两翅上各有几枚飞羽为纯白色, 标 本 1：右侧飞羽 $\mathrm{p} 3 、 \mathrm{p} 4 、 \mathrm{p} 6 、 \mathrm{p} 7 、 \mathrm{p} 8 、 \mathrm{~s} 1 、 \mathrm{~s} 3$ 为纯 白色, 左侧飞羽 $\mathrm{p} 3 、 \mathrm{p} 6 、 \mathrm{p} 7 、 \mathrm{p} 9$ 和 $\mathrm{p} 10$ 纯白色; 标本 2: 右侧飞羽 $\mathrm{p} 5 、 \mathrm{p} 8$ 和 $\mathrm{p} 9$ 为纯白色, 左侧飞 羽 $\mathrm{p} 4, \mathrm{p} 5, \mathrm{p} 7, \mathrm{p} 8, \mathrm{~s} 1, \mathrm{~s} 2$ 为纯白色, 因此在两翅 上形成明显白色条纹。胸暗灰色, 往后逐渐变为棕 灰或暗棕褐色。嘴、脚黑色。特征明显, 野外容易 识别, 形态测量数据见表 1 。

3.1.2 羽色比较 棕色型色彩较鲜艳, 背棕红色, 尾黑色, 外侧尾羽黄褐色。两翅黑色具白色翼斑, 额, 头顶至后颈黑色或灰色, 具黑色贯眼纹; 下体 垓、喉白色, 其余下体棕白色; 飞羽黑色, 初级飞 羽基部白色或棕白色, 形成白色翅斑并明显露出于 覆羽外。黑色型前额、眼先至耳羽、垓、喉、肩及 尾羽、股羽皆黑色, 余部褐灰; 不具翅斑。新色型 与黑色型羽色的主要区别是黑色白边型因为几枚 白色飞羽而形成白色条纹。在标本观察中, 我们还 观察到部分体色介于黑色型和棕色型之间的过渡 类型。

3.1.3 身体量度比较 棕色型与黑色型棕背伯劳 个体间在体长 $\left(F_{1,55}=0.621, P>0.05\right)$ 、体重 $\left(F_{1,55}=0.630, P>0.05\right)$ 、尾长 $\left(F_{1,55}=1.631, P>0.05\right)$ 、 


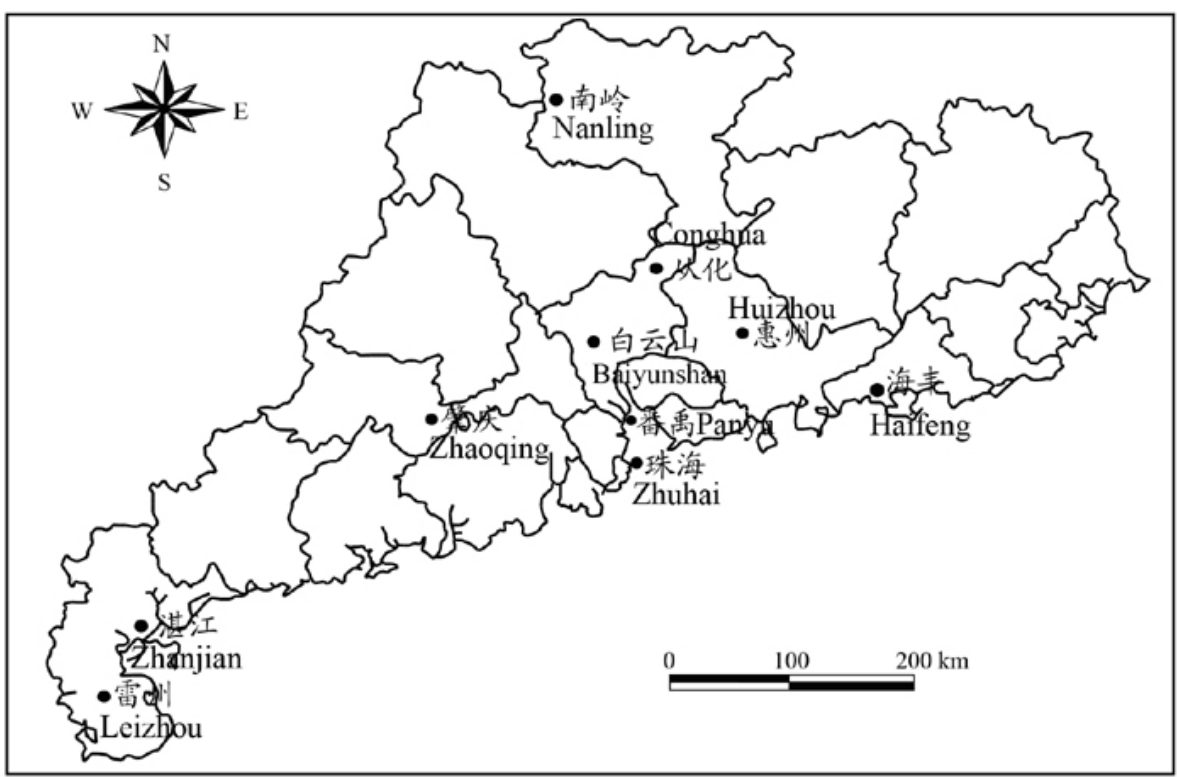

1 样点设置分布示意图

Fig. 1 Demonstration of sampling sites

表 1 棕背伯劳棕色型与黑色型身体量度差异比较

Tab. 1 Body measurements of brown and black morphs of Lanius schach

\begin{tabular}{ccccccc}
\hline \multicolumn{1}{c}{ 色型 } & $\begin{array}{c}\text { 体长 }(\mathrm{mm}) \\
\text { Color morphs }\end{array}$ & $\begin{array}{c}\text { 体重 }(\mathrm{g}) \\
\text { Body length }\end{array}$ & $\begin{array}{c}\text { 尾长 }(\mathrm{mm}) \\
\text { Body weight }\end{array}$ & $\begin{array}{c}\text { Tail length } \\
\text { 嘴峰 }(\mathrm{mm}) \\
\text { Culmen }\end{array}$ & $\begin{array}{c}\text { 翼长 }(\mathrm{mm}) \\
\text { Wing length }\end{array}$ & $\begin{array}{c}\text { 跗蹠 }(\mathrm{mm}) \\
\text { Tarsus }\end{array}$ \\
\hline $\begin{array}{l}\text { 棕色型 }(n=38) \\
\text { Brown morph }\end{array}$ & $256.7 \pm 1.9$ & $59.5 \pm 0.8$ & $128.8 \pm 1.3$ & $17.9 \pm 0.2$ & $108.1 \pm 0.7$ & $32.4 \pm 0.3$ \\
$\begin{array}{l}\text { 黑色型 }(n=19) \\
\text { Black morph }\end{array}$ & $259.5 \pm 3.2$ & $60.8 \pm 1.5$ & $131.7 \pm 1.8$ & $17.4 \pm 0.3$ & $104.6 \pm 1.1$ & $32.9 \pm 0.5$ \\
\hline
\end{tabular}

各组数据在 $P=0.05$ 水平均无显著性差异。

Result indicates no significant differences at $P=0.05$.

嘴峰 $\left(F_{1,55}=1.941, P>0.05\right)$ 、翼长 $\left(F_{1,55}=2.660\right.$, $P>0.05)$ 、跗蹠 $\left(F_{1,55}=1.253, P>0.05\right)$ 上均无显著性 差异 (表 1)。2 个黑色白边型标本量度见表 2（未 作统计分析), 从数据上看与棕色型和黑色型基本 一致。

\section{2 野外种群数量比较}

棕背伯劳棕色型在广东地区种群数量较多, 分 布较为广泛, 在所有的调查点均出现, 且数量达 145; 而其他两种色型种群数量相对较少, 黑色型 共观察到 52 只, 其中大部分见于海丰地区 $(n=46)$, 肇庆、从化和番禺地区也有发现; 黑色白边型最为 稀少, 仅在海丰观察到 3 只。除棕色型外, 其他两
种色型分布范围多集中于海边的部分地区，较多出 现于丘陵区的农田地带, 在广东省海丰县最为集 中, 并且出现 3 种色型在同一地区并存的现象。

\section{4 讨 论}

黑色白边型至今还没有详细资料报道, 武汉大 学收藏的一只 1958 年 2 月 2 日采自广西龙州大青 山的黑伯劳标本, 其左翅第二至第十五枚飞羽和右 翅第二至第十四枚飞羽均为纯白色, Chen et al (1998)认为其为白化现象。本研究表明黑色白边型 身体量度与棕色型及黑色型无显著差异, 出现白色 飞羽的特征也是其特有的, 调查中在同一个区域内

表 2 黑色白边型身体量度

Tab. 2 Body measurements of white-remiged black morphs of Lanius schach

\begin{tabular}{lcccccc}
\hline & $\begin{array}{c}\text { 体长 }(\mathrm{mm}) \\
\text { Stem ength }\end{array}$ & $\begin{array}{c}\text { 体重 }(\mathrm{g}) \\
\text { Body weight }\end{array}$ & $\begin{array}{c}\text { 尾长 }(\mathrm{mm}) \\
\text { Tail length }\end{array}$ & $\begin{array}{c}\text { 嘴峰 }(\mathrm{mm}) \\
\text { Culmen }\end{array}$ & $\begin{array}{c}\text { 翼长 }(\mathrm{mm}) \\
\text { Wing length }\end{array}$ & $\begin{array}{c}\text { 跗蹠 }(\mathrm{mm}) \\
\text { Tarsus }\end{array}$ \\
\hline 标本 1 Specimen 1 & 264.3 & 59.1 & 130.6 & 18.7 & 99.5 & 32.8 \\
标本 2 Specimen 2 & 252.8 & 53.3 & 127.4 & 17.1 & 106.2 & 30.6 \\
\hline
\end{tabular}


共发现 5 个个体(包括 2 个标本), 从其特征及数量 分布认为该类型可能是一种新的色型, 正如多态现 象定义指出的: 一个物种在同一个栖息环境中存在 两个或两个以上完全不同的可遗传形态, 其中在种 群中比例最少的形态不是靠重复突变维持的( $\mathrm{Hu}$ et al, 2007)。

不同色型棕背伯劳身体形态差异主要体现在 羽毛颜色上, 黑色型与棕色型的体色存在显著差 异, 而黑色型与黑色白边型的差别主要体现在翅膀 上是否有具白色飞羽。棕背伯劳除棕色型和黑色型 外, 还存在多种过渡类型, 过渡色型羽色处于棕色 型与黑色型之间呈梯度变化, 在野外不难见到但数 量较少。由于野外观察和分类的困难, 本文未作详 细研究, 这需在以后的研究中加以补充。标本测量 数据表明, 棕色型与黑色型各身体量度没有显著性 差异。本研究在广东省肇庆市永安镇江溪村、海丰 县公平水库观察并拍摄到棕色型与黑色型棕背伯 劳存在相互配对繁殖的现象, 其子代羽色有明显不 同, 黑色型幼鸟羽毛黑褐色, 棕色型幼鸟羽毛棕色

\section{参考文献:}

Baker RR, Parker GA. 1979. The evolution of bird coloration[J]. Phil Trans $R$ Soc Lond Ser B, 287: 63-130.

Butcher GS, Rohwer S. 1989. The evolution of conspicuous and distinctive coloration for communication in birds[J]. Current Ornithology, 6: 51-108.

Chen FG, Luo YS, Zheng GM, Xu WS, Cai QK, Wang YZ, Li DH, Lu JZ. 1998. The Fauna of China: Aves, Vol.9, Passeriformes [M]. Beijing: Science Press, 30-37.[陈服官, 罗有时, 郑光美, 许维枢, 蔡其㑆, 王 延正, 李德浩, 卢济珍. 1998. 中国动物志.鸟纲.第九卷.雀形目. 北 京: 科学出版社, 30-37.]

Endler JA, Thery M. 1996. Interacting effects of lek placement, display behavior, ambient light, and color patterns in three neotropical forest-dwelling birds[J]. Am Nat, 148: 421-452.

Ford EB. 1940. Polymorphism and taxonomy[A]. In: Huxley JS. The New Systematics[M]. Oxford: Clarendon, 493-513.

Galeotti P, Rubolini D, Dunn PO, Fasola M. 2003. Colour polymorphism in birds: Causes and functions[J] . J Evol Biol, 16: 635-646.

Hu HJ, Zeng JY, Liu BY. 2007. Color polymorphism in Birds: Concepts and Hypotheses of Evolutionary Mechanism[J]. Sichuan J Zool, 26(3): 154-158. [胡慧建, 曾今尧, 刘丙万. 2007. 鸟类羽色多态现象: 概念
(另文发表)。表明棕色型和黑色型虽有羽色差别, 但在繁殖上极可能还未产生分离机制, 当然在以后 的调查中还需要进一步观察其杂交后代的可育性 来确认是否存在生殖隔离, 以为研究其分类关系提 供依据。

分化选择假说认为空间和时间的异质性通常 是分化的先决条件（Skúlason \& Smith, 1995; Baker \& Parker, 1979)。广东沿海的部分地区发现多种色型 棕背伯劳并存的现象, 而在广东山地森林分布区 域, 如南岭和连州等地均未观察到黑色型的分布, 表明大生态环境对色型的演化有着重要影响, 多种 色型的产生可能与生境差异有着重要关系。多种色 型并存的现象为什么多集中于沿海边丘陵地区及 近水边的农田地带, 有待于结合不同羽色棕背伯劳 的繁殖生态、繁殖行为以及从分子水平探讨羽色多 态性遗传机制等方面进一步展开。

致谢: 华南濒危动物研究所张春兰、张建新等 在野外调查中提供了很多帮助, 一并表示感谢。

及其进化机制假说. 四川动物, 26(3): 154-158.]

Huxley J. 1955. Morphism in Birds[C]. Acta Int Congr Ornithol, XI: 309-328.

Marchetti K. 1993. Dark habitats and bright birds illustrate the role of the environment in species divergence[J]. Nature, 362: 149-152.

Mather K. 1955. Polymorphism as an outcome of disruptive selection [J]. Evolution, 9: 52-61.

Rohwer S, Paulson DR. 1987. The avoidance-image hypothesis and color polymorphism in Buteo hawks[J]. Orn Scand, 18: 285-290.

Skúlason S, Smith TB. 1995. Resource polymorphisms in vertebrates[J]. Trends Ecol Evol, 10: 366-370.

Zheng ZX. 1976. A Synopsis of the Avifauna of China. 2ed edition [M]. Beijing: Science Press, 460-470. [郑作新. 1976. 中国鸟类分布名录 (第二版). 北京: 科学出版社, 460-470.]

Zheng GM. 2005. A Classification and Distribution of the Birds of China[M]. Beijing: Science Press. [郑光美. 2005. 中国鸟类分类与分 布名录. 北京: 科学出版社.]

Zhao ZJ. 2001. A Handbook of the Birds of China[M]. Changchun: Jilin Science and Technology Press, 128-146. [赵正阶. 2001. 中国鸟类 志·卷. 长春: 吉林科学技术出版社, 128-146.] 